\title{
Evaluation of an automatic platelet counting system utilizing whole blood
}

\author{
R. M. ROWAN, W. Allan, AND R. J. PRESCOtT \\ From the Department of Haematology, Royal Infirmary, Edinburgh
}

SYNOPSIS Technicon's newly developed platelet AutoCounter utilizes an electro-opticalsystem which detects and counts particles by sensing the scattering of light which occurs when blood cells flow through the illuminated sensing chamber of a micro-optical system. The system utilizes whole blood collected into EDTA. Blood samples, which can be handled at the rate of 40 per hour, are diluted with $2 \mathrm{M}$ urea which in addition causes lysis of the erythrocytes. The haemolysate thus obtained is delivered to the particle counter after a two-minute reaction time, the cell count finally being displayed on a continuous chart recorder.

An evaluation of this machine has been carried out in the Department of Haematology, the Royal Infirmary, Edinburgh, on hospital specimens from patients suffering from a wide variety of diseases. The results of this evaluation are presented. Reproducibility studies indicate a coefficient of variation of $4 \%$ at any platelet level. The percentage drift per sample ranges from +0.4 to $-0.4 \%$ of the total count. Carry-over and departures from linearity are statistically significant; however, the magnitude of these deviations is not sufficient to cause concern in routine use. Acceptable agreement is noted between machine counts and the counts obtained by technicians using phasecontrast microscopy with the exception of two anomalous individuals.

Discrepant counts were noted in patients with elevation of the erythrocyte sedimentation rate and also in patients with disturbance of immunoglobulins. A modification to the sampling probe which eliminates the former problem is described.

The AutoCounter described in this paper provides a fast, reliable, and accurate service laboratory platelet counting system.

Morphological and numerical evaluation of formed blood elements constitutes useful guidance in the assessment of normal and disturbed haemopoietic function. Because of increasing interest in automation of the haematology laboratory there is need for a parallel increase in cooperation between the haematologist and the automated instrument designer in order that each may gain insight into the other's problems. Already automation procedures in haematology are well established with respect to red and white cell counting (Nelson, 1969; Barnard, Carter, Crosland-Taylor, and Stewart, 1969) but this satisfactory state does not yet extend to enumeration of platelets, although the clinical usefulness of platelet counts is irrefutable.

The accuracy of platelet counting techniques is far from satisfactory. In 1937 Tocantins evaluated 116 different methods of platelet counting. Since then Received for publication 8 December 1971. modifications have been made to older methods and additional newer methods have been introduced so that the number of techniques now available exceeds 160 (Maupin, 1969). This proliferation of methods proves the inadequacy of available techniques and also stresses the need for improved systems.

Direct counting methods involve the absolute enumeration of platelets in a suitable solution and thus the platelet count is not related to a second known quantity of formed elements as was the practice with now discarded indirect methods of platelet counting. Examples of direct counting methods in current use include (1) light or phasecontrast microscopy techniques, (2) electronic particle counting which detects and counts cells by sensing a change in the electrical conductivity of a channel separating two electrolyte solutions when blood cells flow through this channel, and (3) the electro-optical method of counting which detects 
and counts cells by sensing the scattering of light which occurs when blood cells flow through the illuminated sensing chamber of a micro-optical system.

These latter techniques have certain inherent limitations which, depending on the effectiveness of instrument design, affect the ultimate accuracy and reproducibility of the blood cell counting system. Electronic particle counting systems have been considered in a number of recent reviews (Sipe and Cronkite, 1962; Eggleton and Sharpe, 1963; Bull, Schneiderman, and Brecher, 1965). Even by such sophisticated means, however, platelet counts are difficult to accomplish reliably. The main problems encountered include unsatisfactory separation of red cells from platelet-containing plasma; the tendency of platelets to aggregate; and the presence in the diluent of particles which are only little smaller than platelets and which are not excluded by the threshold settings employed. Furthermore, significant errors may arise from the instrument itself and its calibration.
The purpose of this paper is to present the results of an evaluation of the Technicon platelet AutoCounter carried out in the Department of Haematology, the Royal Infirmary, Edinburgh. The Technicon system is an electro-optical system utilizing the light-scattering principle.

\section{The AutoCounter System}

The Technicon platelet AutoCounter is fully automated to quantitate platelets rapidly from whole blood without prior manipulation. One operator can handle the analytical train and the system delivers platelet counts at the rate of $\mathbf{4 0}$ per hour.

The five main components of the system are illustrated in Figure 1. The sampler module consists of a removable circular tray with spaces for $\mathbf{4 0}$ sample containers. The blood samples are held in uniform suspension by rotating Teflon paddles. A probe automatically dips into each sample cup and aspirates $0.4 \mathrm{ml}$ whole blood. A proportioning pump continuously propels diluting fluids and samples at

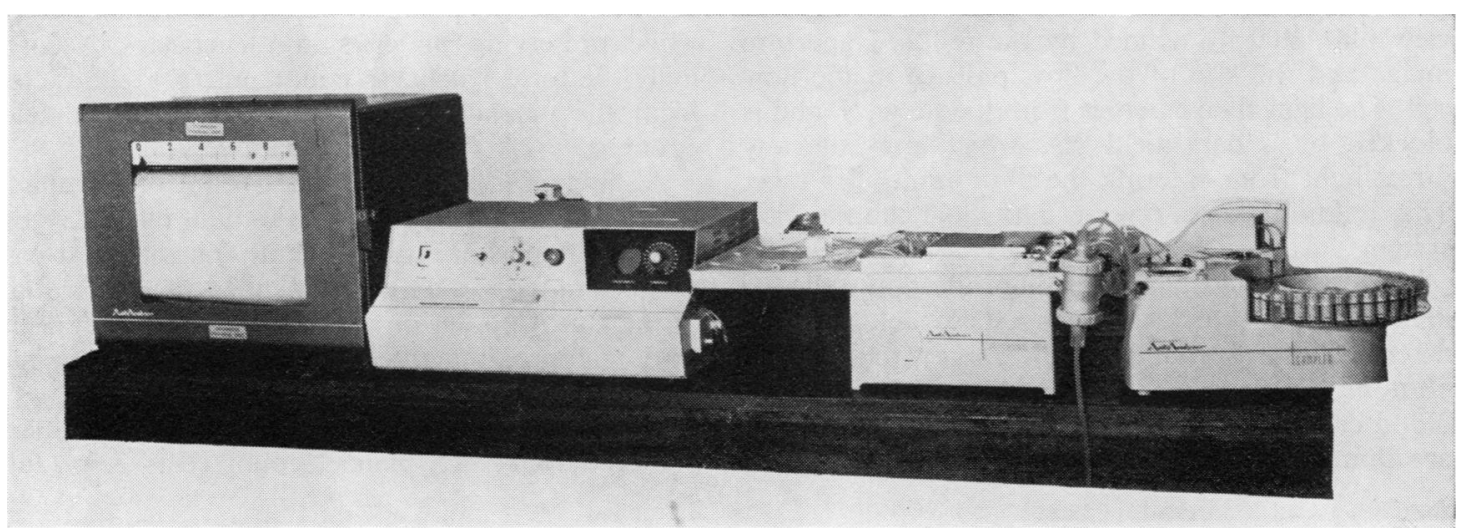

Fig. 1 The AutoCounter system.

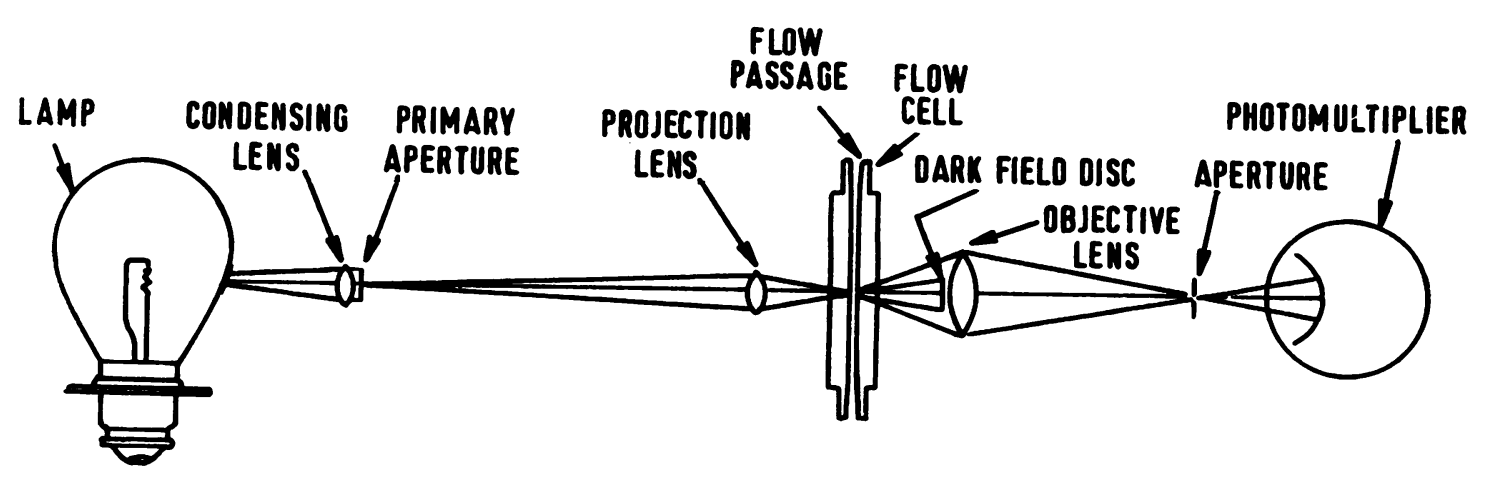

Fig. 2 The optical system (by courtesy of the Technicon Corporation). 
precisely determined flow rates through a closed flow system of plastic and glass tubing (manifold), in which samples and reagents are brought together under constant physical conditions. The first dilution in the manifold, as determined by the proportioning pump and the inside diameter of the manifold, is a 1:21 dilution and the final dilution eventually delivered for counting is 1:1500. Following aspiration of each sample, a small bubble of air is aspirated, followed by wash fluid, followed in turn by another bubble of air, and then the sequence repeats itself. The air segments provide an antidiffusion barrier between sample and wash fluid and the next mixed sample.

The cell counter uses an optical system (Fig. 2) to generate light pulses from platelets passing through the flow cell. Light from the lamp passes through a condensing lens, uniformly illuminating the primary aperture. The primary aperture has a small rectangular opening in its centre. A projection lens picks up the light coming through this aperture and forms a reduced image of the aperture in the centre of the flow cell. This results in a small, brightly illuminated view film, sharply defined by the reduced aperture image and the size of the flow passage in the flow cell. The light then emerges from the flow cell and is blocked by a dark field disc which prevents any direct light from entering the photomultiplier tube. This is known as a reverse dark field illumination system.

If a clear liquid passes through the flow cell, the light rays are blocked by the dark field disc as described above, and no light energy reaches the photomultiplier tube; however, if particles, such as blood cells, are dispersed in the liquid stream, they are illuminated as they pass through the view volume.

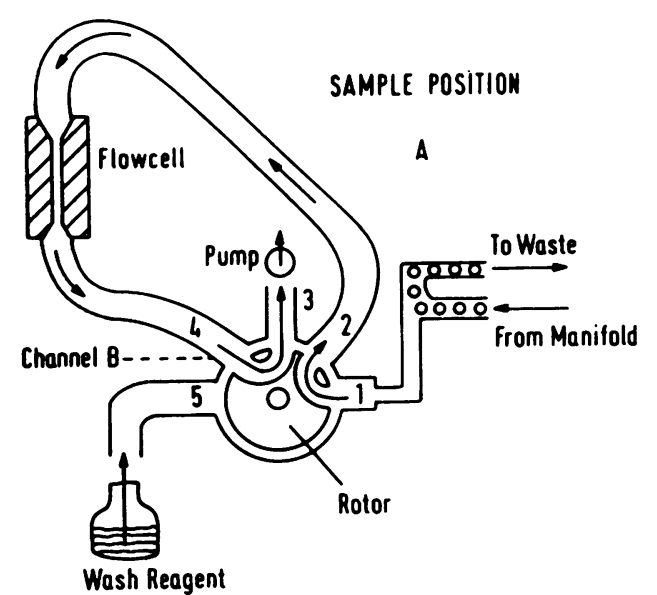

When illuminated, the particles become secondary sources of illumination, scattering light in a forward direction towards the objective lens. The objective lens collects this light and focuses it through a small aperture in front of the photomultiplier tube. The aperture blocks out stray light, allowing only the scattered light from the particle to enter the photomultiplier tube. Thus, as each particle passes through the view volume, an electrical pulse is generated. These pulses can be viewed on an oscilloscope screen located on the front panel. The light pulses, once transformed into electrical pulses, are amplified and counted in an electrical system finally being displayed on chart paper.

Figure 3 shows the cell counter wash valve. In the sample position (Fig. 3A) the diluted haemolysate flows through the flow cell from above downwards. A rotating valve permits flow of a sodium hydroxide/ tergitol mixture in the reverse direction (Fig. 3B) to cleanse thoroughly the narrow channel of the flow cell between each sample.

The diluent, which consists of a two-molar urea solution, acts in addition as an haemolysing agent while preserving platelets and leucocytes. Accordingly the total leucocyte count must be subtracted from the machine result before issuing a platelet count.

A standard is provided by the Technicon Company which consists of preserved human platelets suspended in plasma. Initially both normal and low range standards were recommended but calibration of the AutoCounter on a single standard of concentration approximately 300000 platelets $/ \mathrm{c} \mathrm{mm}$ has proved completely satisfactory.

Using materials recommended by the instrument manufacturers each platelet count costs $3 \cdot 6 \mathrm{p}$ (July

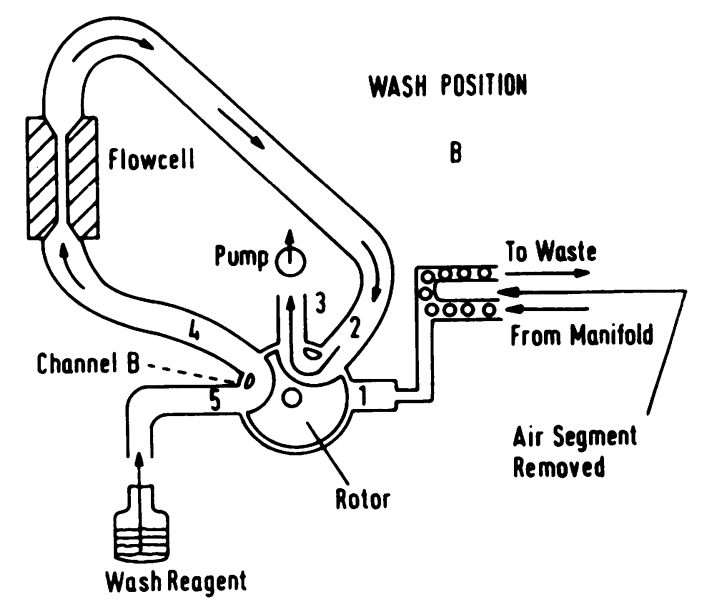

Fig. 3 The cell counter wash valve (by courtesy of the Technicon Corporation). 
1971). This figure does not take into account the purchase or depreciation of the machine, nor does it include servicing costs.

\section{Materials and Methods}

The haematology laboratory of a large teaching hospital was the site of this evaluation, specimens coming from patients with a wide variety of diseases.

Blood was withdrawn into dry plastic syringes using a $21 \mathrm{G}$ needle taking care that the venepuncture was a clean one and that the blood flowed into the syringe spontaneously without frothing. The needle was then detached from the syringe and the requisite amount of blood delivered without frothing into an appropriate vessel containing dipotassium EDTA giving a final EDTA/blood concentration of $1 \mathrm{mg} /$ ml. Blood and anticoagulant were gently mixed without delay. All samples were processed within two hours.

The visual direct counting technique using the formal-citrate red-cell diluent described by Dacie and Lewis (1970) was used. Platelets were counted using phase-contrast microscopy. The number of platelets in a minimum of $4 \mathrm{~mm}^{2}$ on a Neubauer chamber was counted. Whenever duplicate or triplicate counts were made, they were true replicates and performed by different technicians.

The AutoCounter was set up according to the manufacturer's recommendations. Optical focusing is critical, but once aligned the system proved to be stable.

It is important to wash the AutoCounter manifold thoroughly twice daily using a sodium hydroxide/ tergitol wash solution. The diluent filter (Whatman's grade 03 filter candle) proved entirely satisfactory provided it was not permitted to dry out when urea would crystallize. The filter requires changing when insufficient diluent is being drawn through the system. Such an occurrence should be considered when there is any upset in the manifold bubble pattern.

\section{Results and Discussion}

\section{REPRODUCIBILITY AND DRIFT}

The effect of platelet levels on the reproducibility of the AutoCounter was examined with a series of duplicate counts on 112 blood samples which were obtained over a period of six days. On each day the procedure was to count the complete batch and then to re-count the samples. As no adjustments were made to the AutoCounter in this time, the differences between pairs of observations on the same sample were due to the variability of the machine with a given sample and to drifting of the machine before resampling. For each day's run the mean proportionate change between pairs of observations on the same blood was determined and a correction based on this mean change was applied to the second reading from each pair. The corrected difference is plotted against the corrected average in Figure 4. A linear regression of the difference on the average gave a small negative intercept on the $y$ axis which did not differ significantly from zero. Accordingly a regression through the origin was performed and is also shown in Figure 4. Thus, over the range encountered in this series, differences of approximately $5 \%$ of the average value would be expected in pairs of observations. Alternatively expressed, the coefficient of variation at any platelet level is approximately $4 \%$.

This experiment also enabled the rate of drift to be calculated for each day's set of observations, using the mean proportionate change between counts on the same blood as the basis for the calculation. The rates of drift on the six days, expressed as the percentage drift per sample, were $-0.3 \%,-0.1 \%$, $-0.4 \%,-0.4 \%,+0.4 \%$, and $0.0 \%$.

The measure of variability in duplicate counts at different platelet levels, as described above, is useful in demonstrating the relationship between reproducibility and platelet level but as an estimate of reproducibility error in routine use it will probably give overestimates, since it is based on long periods of using the machine without readjustment. A smaller series of six bloods throughout a range of 30000 to 600000 platelets which were successively resampled

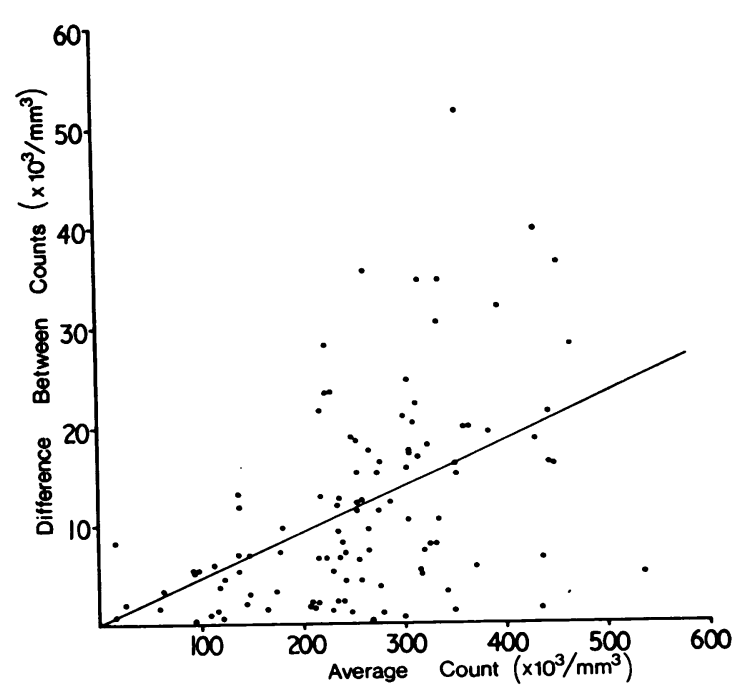

Fig. 4 Reproducibility experiment showing the corrected difference plotted against the corrected average. 
at least 12 times gave standard deviations which were approximately half the size of those predicted above (Table I).

\begin{tabular}{lllllll}
\hline Blood No. & \multicolumn{7}{l}{ Platelet Count $\times 10^{2} \mathrm{~mm}^{3}$} \\
\hline 1 & 35 & 115 & 220 & 315 & 460 & 660 \\
2 & 35 & 110 & 225 & 315 & 460 & 650 \\
3 & 35 & 115 & 220 & 320 & 460 & 670 \\
4 & 30 & 100 & 220 & 310 & 470 & 650 \\
5 & 30 & 100 & 225 & 315 & 470 & 645 \\
6 & 30 & 105 & 230 & 310 & 465 & 650 \\
7 & 30 & 105 & 220 & 305 & 465 & 665 \\
8 & 30 & 105 & 230 & 310 & 465 & 660 \\
9 & 30 & 105 & 230 & 305 & 470 & 660 \\
10 & 30 & 105 & 225 & 305 & 460 & 650 \\
11 & 30 & 110 & 235 & 310 & 460 & 650 \\
12 & 30 & 105 & 220 & 320 & 460 & 660 \\
\hline
\end{tabular}

Table I Replicate studies on a series of six bloods successively resampled 12 times

\section{LINEARITY AND CARRY-OVER}

Four hundred $\mathrm{ml}$ of whole blood was withdrawn from a normal blood donor into sequestrene giving a final concentration of $1 \mathrm{mg} / \mathrm{ml}$. This was subsequently manipulated by differential centrifugation to give platelet-rich plasma to which red blood cells were then added in sufficient volume to produce a PCV within normal limits $(40 \%)$. This manipulated whole blood sample, which had a final platelet count of 650000 , was then diluted with normal saline to give $80 \%, 60 \%, 40 \%, 20 \%$, and $10 \%$ dilutions. Normal saline was used as a zero control. Each of those seven dilutions was subsequently divided into seven separate aliquots. The 49 samples thus obtained were then counted on the AutoCounter. The order of presentation was carefully controlled to ensure that the seven samples at each dilution were immediately preceded by one sample at each of the seven dilutions.

When examining the results it was clear that the machine had drifted, and a correction was applied assuming a constant proportional drift throughout the duration of the experiment. The size of this drift was estimated in such a way that the error sum of squares was minimized in the subsequent analysis, and the magnitude of the drift was found to be $0 \cdot 1 \%$ per observation.
A further factor influencing the analysis was the unequal variability at different dilutions. In order to give an appropriate weight to each reading; the variance of the observations at each dilution was calculated and a linear regression of variance on dilution was performed. The weighting factors in the following analysis were then taken to be proportional to the reciprocal of the fitted variances at each dilution.

The design of the experiment enabled it to be treated in the first instance as a randomized completeblock design and an analysis of variance determined the sums of squares attributable to experimental error, carry-over, and differing dilutions. The sums of squares attributable to dilutions were further subdivided into that due to a linear association between the count and dilution and that due to deviations from linearity.

Table II presents the analysis of variance, and it is seen that both carry-over and departures from linearity are statistically significant. However, both of these effects are small and a less detailed experiment would have been unlikely to detect them. If we assume no carry-over at the $0 \%$ dilution, the observed carry-over at the $100,80,60,40,20$, and $10 \%$ dilutions are $2 \cdot 1,5 \cdot 1,2 \cdot 4,4 \cdot 8,2 \cdot 7$, and $2 \cdot 6 \times 10^{3}$

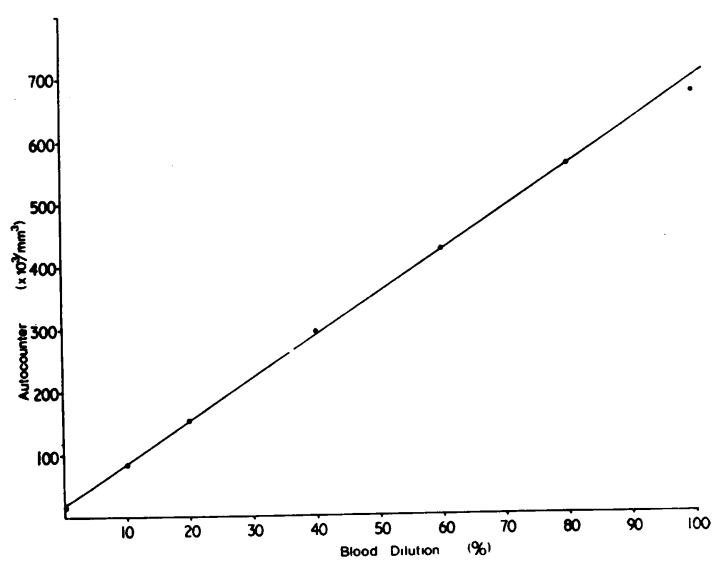

Fig. 5 Linearity experiment.

\begin{tabular}{lcccc}
\hline Source of Variation & $d f$ & Sums of Squares & Mean Square & $F$ \\
\hline Linearity with dilution & 1 & 34754035 & & $34 \cdot 6(\mathrm{p}<<0.001)$ \\
Deviations from linearity & 5 & 24724 & 4945 & $2 \cdot 5(\mathrm{p}<0.05)$ \\
Carry-over & 6 & 2103 & $305 \cdot 5$ & 142.9 \\
Experimental error & 35 & 3002 & & \\
Total $^{1}$ & 47 & 34785864 & & \\
\hline
\end{tabular}

Table II Analysis of variance

${ }^{1}$ The degrees of freedom for these entries have been reduced by 1 from their normal values to allow for the drift correction which has been applied. 
counts $/ \mathrm{mm}^{3}$, respectively. The non-linearity is shown in Figure 5. It is evident that agreement is good over the lower part of the range, with a tendency to count low at high platelet concentrations. The magnitude of this deviation would not be sufficient, however, to cause concern in routine use.

AGREEMENT WITH PHASE-CONTRAST COUNTS Two series of blood were counted by experienced technicians using the phase-contrast method and using the automatic counter. The white blood count was obtained separately using the Coulter model S. As it was known that AutoCounter results from patients with myelomatosis may be unreliable (Widmark and Weschler, 1970), such bloods were not included in the series. However, patients with non-paraprotein ESR elevation were included to see if the high ESR as such was instrumental in causing the discrepant counts. Figures 6 and 7 show the plot of the machine count against the combined platelet and WBC count obtained by technicians. Those samples with high ESRs ( $>20 \mathrm{~mm}$ ) are shown separately from those with normal ESRs. In both series a statistical comparison of the linear regression lines, obtained separately for low and high ESR bloods (by means of a weighted analysis of covariance), showed no significant difference in slope, intercept, or scatter about the fitted regression lines $(P>0.05)$. The lines shown in Figs. 6 and 7 give the best weighted least squares fit, obtained from pooling all points, irrespective of the ESR.

In both series there is generally reasonable agreement to the regression line though each series has one wild point whose distance from the regression line is greater than that expected by random variation. There is no apparent common factor between them, one blood having a high ESR and the other a normal ESR. As these points differ significantly from the regression lines and would have had an undue effect on the remainder of the statistical analysis, they were not included in the analysis previously described.

COMPARISON OF LINEARITY WITH AUTOCOUNTER AND PHASE-CONTRAST METHODS

Three bloods were prepared at differing dilutions and each sample was counted on the AutoCounter and by two technicians using the phase-contrast method. Figure 8 shows the results of the AutoCounter and the average of the two technicians. The individual counts of the two technicians were generally in extremely close agreement, and never differed by more than $38 \times 10^{3}$ counts $/ \mathrm{mm}^{3}$. The lines in Fig. 8 show the best fitting line through the origin for the autocounts. For each blood the linearity is seen to be good. The manual counts also

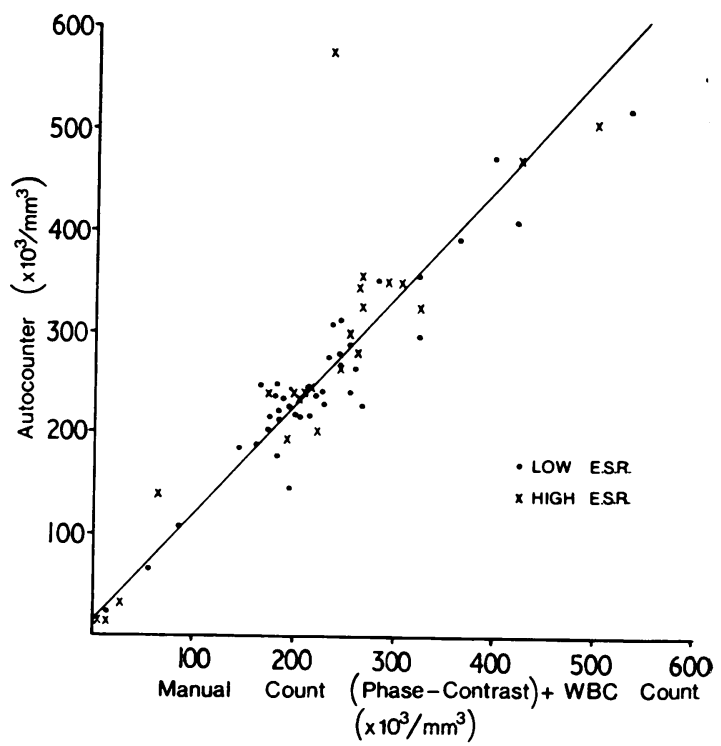

Fig. 6 Machine count plotted against combined platelet and white cell count obtained by technicians.

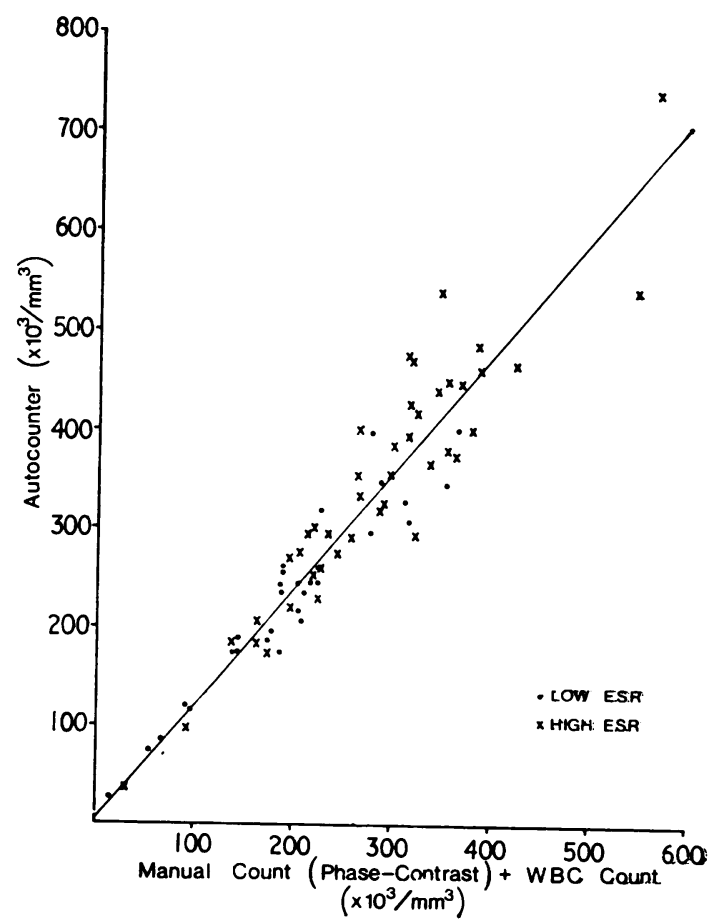

Fig. 7 Machine count plotted against combined platelet and white cell count obtained by technicians. 


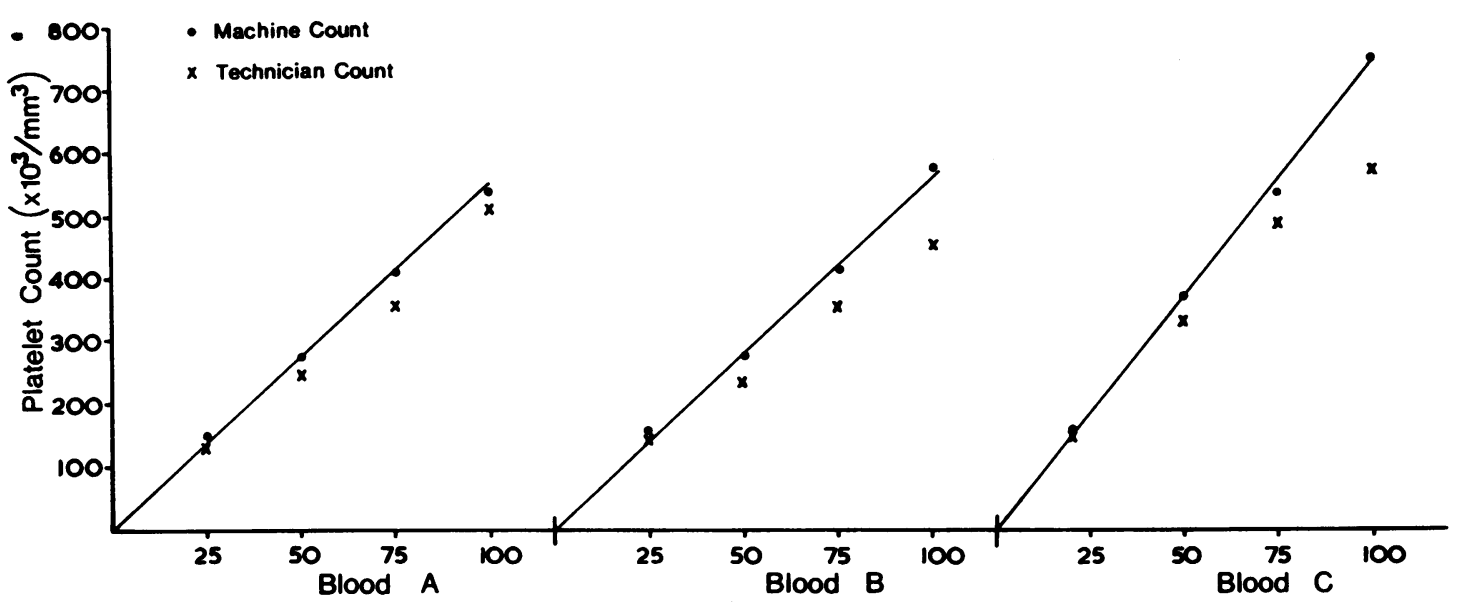

Dilution \%

Fig. 8 Experiments comparing linearity with the AutoCounter and the phase-contrast microscopy method.

show reasonable linearity for bloods $A$ and $B$. However, for blood C, which was manipulated to give a particularly high platelet count at $100 \%$ dilution, there is a noticeable non-linearity, taking the form of a low count at the higher platelet concentrations.

\section{Discussion}

Evaluation of the platelet AutoCounter system has brought to light certain conditions which substantially alter the automatic counts. Bloods with a high ESR produce a peaking phenomenon on the tracing (Fig. 9) which, although not causing seriously discrepant results, may in fact lead to difficulty in reading the result. The only consistent abnormality in these bloods was marked elevation of fibrinogen levels. Protein electrophoresis on cellulose acetate failed to demonstrate any consistent or significant anomaly. Substitution of patient's plasma with either physiological saline or ABO compatible normal plasma removed this 'peaking' phenomenon. It was concluded that 'peaking' was produced by sedimentary tailing akin to erythrocyte sedimentation in the sample as it passed through the diluting system. This was in fact visible on examination of the red cells in the sample at various points in the manifold. In the counting chamber a greater proportion of particles presented in the last third of the sample but the increase was not linear and the problem could not be cured by taking the mean count. Accordingly a modified sampling probe was constructed which permitted air segmented sampling

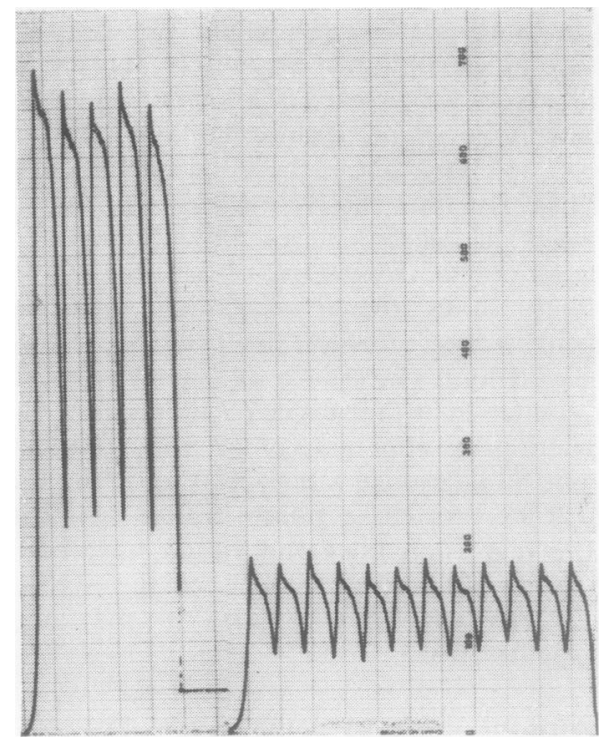

Fig. 9 Peaking phenomenon produced by bloods with a high ESR.

instead of continuous blood sampling. This removed the problem of peaking (Figs. 10 and 11).

It has been previously reported by Widmark and Weschler (1970) that patients with abnormally high serum proteins, such as paraprotein disturbances and red cell autoantibody disorders, may cause 'offscale' results. This has been our experience in that in addition to 'peaking', serious discrepancies have 


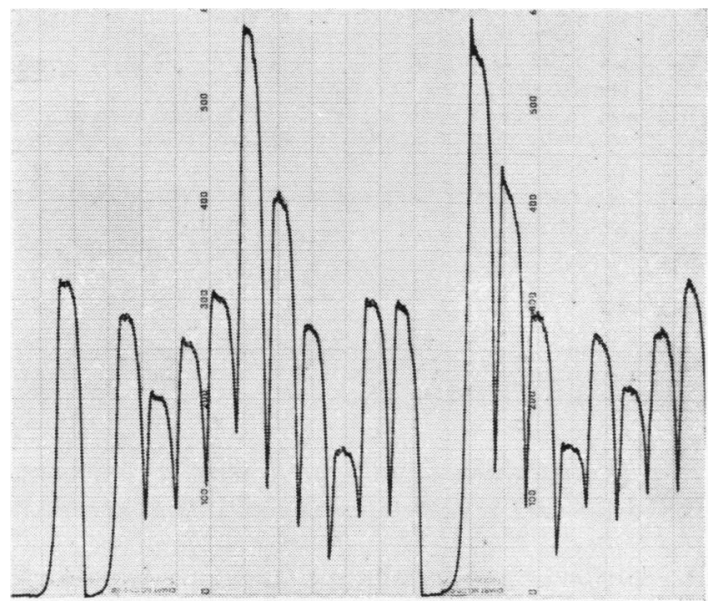

Fig. 10 Two series of counts. Those counts on the righthand side of the illustration show peaking produced by bloods with a high ESR. The tracings on the left-hand side show correction of this phenomenon following the incorporation of air segmented sampling.

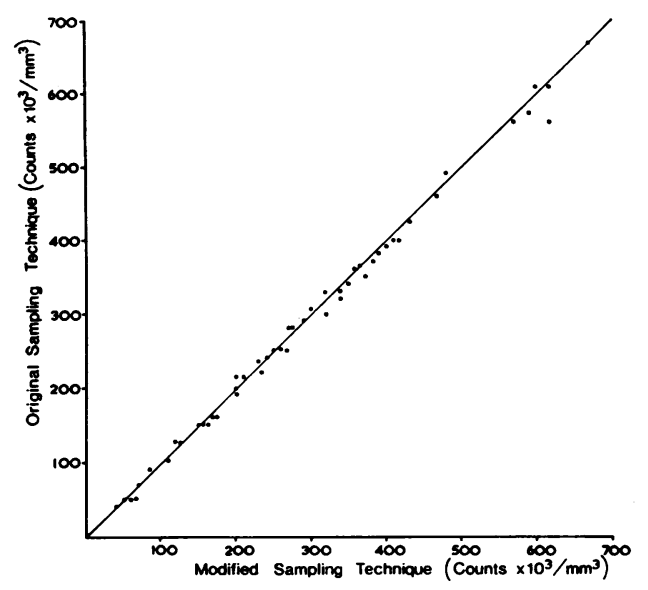

Fig. 11 Counts using the original sampling technique plotted against those obtained with the modified sampling technique.

been demonstrated between automatic counts and visual counts in patients with paraprotein disorders. In a series of 10 patients with paraprotein disturbances (mainly IgG) six gave an acceptable correlation between automatic and visual counts, the remainder giving totally unacceptable results, the machine results being two to three times higher than the visual count. One patient with myelomatosis and an IgG level of $8 \mathrm{~g} / 100 \mathrm{ml}$ gave counts of 412000 on the AutoCounter and 208000 by phase- contrast methods. On the other hand, a second patient, also suffering from myelomatosis, with an IgG content of $9.6 \mathrm{~g}$, gave counts of 62500 on the machine and 47000 by phase-contrast microscopy. Obviously the degree of variation between counts is not related to the quantity of immunoglobulin present. Although the exact cause of this discrepancy remains conjectural, it is possible that coating of subthreshold fragments, possibly red cell stroma, by the abnormal protein may increase the number of threshold particles presented to the counting cell. This abnormality is not corrected by the sampling modification applied to the AutoCounter. Peaks produced in this situation are often 'off-scale'. Saline dilution experiments give platelet counts which are not linear, in contradistinction to true thrombocytoses. Instances in which such problems are encountered include any condition with abnormally high serum proteins and/or erythrocyte autoantibodies. Non-linearity of diluted samples should serve as an indication to check the level of plasma proteins and to perform a direct antiglobulin test.

Further problems may arise in situations where there is a high total white count, numerous nucleated red cells, Howell-Jolly bodies, or malarial parasites, all of which provide particles countable by the system. It should also be remembered that platelet autoantibodies may produce a low platelet count as recorded by the counter due to platelet agglutination. Unfortunately such cases did not arise during the period of the survey, but it is clear that such conditions can be detected by careful scrutiny of peripheral blood films.

In conclusion, this fully automated platelet counting system, although not fully satisfying stringent conditions of linearity and carry-over, gives results which are totally acceptable in terms of service laboratory platelet counting. An important advantage is that the system processes whole blood samples without prior manipulation at the rate of $\mathbf{4 0}$ samples per hour.

We wish to thank the Technicon Corporation for providing the AutoCounter on which the evaluation was carried out. We are grateful to Dr S. H. Davies, Department of Haematology, the Royal Infirmary, Edinburgh, for encouragement and advice.

\section{References}

Barnard, D. F., Carter, A. B., Crosland-Taylor, P. J., and Stewart, J. W. (1969). An evaluation of the Coulter Model S. J. clin. Path., 22, Suppl. (Coll. Path.), 3, 26-33.

Bull, B. S., Schneiderman, M. A., and Brecher, G. (1965). Platelet counts with the Coulter counter. Amer. J. clin. Path., 44, 678688.

Dacie, J. V., and Lewis, S M. (1970). In Practical Haematology, 4th. ed., p. 70. Churchill, London. 
Eggleton, M. J., and Sharpe, A. A. (1963). Platelet counting using the Coulter electronic counter. J. clin. Path., 16, 164-167.

Maupin, B. (1969). In Blood Platelets in Man and Animals, vol. 1, p. 37. Pergamon, Oxford.

Nelson, M. G. (1969). Multichannel continuous flow analysis on the SMA-4/-7A. J. clin. Path., 22, Suppl. (Coll. Path.), 3, 20-25.

Sipe, C. R., and Cronkite, E. P. (1962). Studies on the application of the Coulter electronic counter in enumeration of platelets. Ann. N.Y. Acad. Sci., 99, 262-270.

Tocantins, L. M. (1937). Technical methods for the study of blood platelets. Arch. Path., 23, 850-879.

Widmark, R. M., and Weschler, W. A. (1970). Evaluation of a fully automatic platelet counting technique. Technicon Quarterly, 2, 23-28.

\section{The February 1972 Issue}

\section{THE FEBRUARY 1972 ISSUE CONTAINS THE FOLLOWING PAPERS}

Ischaemic lesions of the alimentary tract G. S. A. MCDONALD AND D. O'B. HOURIHANE

Hilar cell tumour of the ovary M. C. ANDERSON

The diagnosis of trophoblastic tumours from uterine curettings $\quad$ c. W. ELSTON AND K. D. BAGSHAWE

The structure of the breast in mucoviscidosis A. MILFORD WARD

The value of a study of the mucosubstances in rectal biopsies from patients with carcinoma of the rectum and lower sigmoid in the diagnosis of premalignant mucosa M. ISABEL FILIPE

Evaluation of the Lundh test in the diagnosis of pancreatic disease G. E. LEVIN, G. R. YOUNGS, AND I. A. D. BOUCHIER

Measurements of concentrations of human serum immunoglobulins

Immunoglobulin levels in infantile pneumocystosis ELFRIEDE KOHOUT, CORNELIUS POST, BAHRAM AZADEH, WERNER DUTZ, BASHI BANDARIZADEH, AND DARIUS KADIVAR

Serum hydroxybutyrate dehydrogenase (HBD) assays in the clinical laboratory $K$. MONTAZEMI AND J. G. LINES

The nature of the alkaline phosphatases of bile C. P. PRICE, P. G. HILL, AND H. G. SAMMONS

Clinical application and mechanization of a simple and inexpensive method for estimating $T_{3}$ uptake in serum W. W. WALTHER
Haem biosynthesis studied in patients with rheumatoid arthritis s. v. JOHANSSON AND P. O. STRANDBERG A satisfactory quantitative test of lymphocyte response to phytohaemagglutinin for the definition of normal control values and recognition of immunological defects MARGARET G. FITZGERALD

Target cells in haemoglobinopathies L. R. DAVIS

Microbiological assay of amino acids in serum: valine, leucine, and methionine J. A. BLACKMORE AND T. E. PARRY

Changes in antibiotic sensitivity patterns of Gramnegative bacilli in burns ELIZABETH ROE AND E. J. L. LOWBURY

The isolation of an X-dependent strain of Haemophilus from otitis media identified as $H$. haemoglobinophilus (canis) JOYCE FRAZER AND K. B. ROGERS

\section{Technical method}

The effect of autoclaving characteristics on the recovery of serum vitamin $\mathbf{B}_{\mathbf{1 2}}$ determined by a radioisotope dilution method $D$. $H$. ORRELL AND A. D. CASWELL

Letter to the Editor

Book reviews

Notices

Copies are still available and may be obtained from the PUBLISHING MANAGER, BRITISH MEDICAL ASSOCIATION, TAVISTOCK SQUARE, LONDON, WClH 9JR, price, $£ 1.05$ 